\title{
ERRATUM
}

\section{Cognitively Relevant Recoding in Hippocampus: Beneficial Feedback of Ensemble Codes in a Closed Loop Paradigm}

\author{
Robert E. Hampson, John D. Simeral, Theodore W. Berger, Dong Song, \\ Rosa H. M. Chan, Vasilis Z. Marmarelis, and Sam A. Deadwyler
}

Robert P. Vertes and Robert W. Stackman Jr. (eds.), Electrophysiological Recording Techniques, Neuromethods, vol. 54, DOI 10.1007/978-1-60327-202-5, (C) Springer Science+Business Media, LLC 2011

DOI 10.1007/978-1-60327-202-5_9

The publisher regrets that in the print and online versions of this book the metadata for the title of the chapter entitled "Cognitively Relevant Recoding in Hippocampus: Beneficial Feedback of Ensemble Codes in a Closed Loop Paradigm", by Robert E. Hampson, John D. Simeral, Theodore W. Berger, Dong Song, Rosa H. M. Chan, Vasilis Z. Marmarelis, and Sam A. Deadwyler is incorrect. The correct title is "Cognitively Relevant Recording in Hippocampus: Beneficial Feedback of Ensemble Codes in a Closed Loop Paradigm". 